\title{
Prognostic value of ERG, PTEN, CRISP3 and SPINK1 in predicting biochemical recurrence in prostate cancer
}

\author{
BYEONG-JOO NOH ${ }^{1}$, JI-YOUN SUNG ${ }^{1,2}$, YOUN WHA KIM ${ }^{1,2}$, SUNG-GOO CHANG ${ }^{3}$ and YONG-KOO PARK ${ }^{1,2}$ \\ ${ }^{1}$ Department of Medicine, Graduate School, Kyung Hee University; Departments of ${ }^{2}$ Pathology and ${ }^{3}$ Urology, \\ Kyung Hee University Hospital, Seoul 02447, Republic of Korea
}

Received March 2, 2015; Accepted March 15, 2016

DOI: $10.3892 / \mathrm{ol} .2016 .4459$

\begin{abstract}
The established prognostic factors associated with prostatic adenocarcinoma are the Gleason score, pathological $\mathrm{T}$ staging and serum prostatic-specific antigen (PSA) level. However, these prognostic factors alone are not sufficient for predicting prognostic characteristics, including early stage or advanced prostate cancer, presence of metastasis or disease-related mortality. The purpose of the present study was to simultaneously evaluate the prognostic value and associations of four biomarkers, namely, transcriptional regulator ERG (ERG), phosphatase and tensin homolog (PTEN), cysteine-rich secretory protein 3 (CRISP3) and serine protease inhibitor Kazal type I (SPINK1), and to conduct risk stratification of prostate cancer for use in patient management. A total of 68 formalin-fixed, paraffin-embedded, prostate cancer samples from radical prostatectomies were obtained in the Kyung Hee University Hospital (Seoul, Korea) and were studied immunohistochemically for ERG, PTEN, CRISP3 and SPINK1 to determine the proportion and intensity of staining. SPINK1 expression was mutually exclusive of ERG expression $(\mathrm{P}=0.001)$. The loss of PTEN and high CRISP3 expression are unfavorable indicators for prostate cancer, as PTEN loss was associated with shorter biochemical recurrence $(\mathrm{BCR})(\mathrm{P}=0.039)$, and high CRISP3 expression was associated with increased $\mathrm{BCR}(\mathrm{P}<0.001)$ and cancer-related mortalities $(\mathrm{P}=0.011)$. Using the combination of low PTEN and high CRISP3 expression enables attention to be focused on patients who exhibit a poor prognosis. Subgrouping of patients, into high-risk and low-risk categories, was correlated with BCR-free survival in prostate cancer upon multivariate analysis $(\mathrm{P}=0.030)$. Overall, low
\end{abstract}

Correspondence to: Professor Yong-Koo Park, Department of Medicine, Graduate School, Kyung Hee University, 23 Kyungheedaero, Dongdaemun, Seoul 02447, Republic of Korea E-mail: ykpark@khmc.or.kr

Key words: prostate cancer, cysteine-rich secretory protein 3, phosphatase and tensin homolog, serine protease inhibitor Kazal type I, transcriptional regulator ERG
PTEN and high CRISP3 expression significantly characterize the subgroups of prostate cancer that have a poor prognosis for BCR.

\section{Introduction}

Prostate cancer is the fifth most common malignancy in Korea (1), but the second most prevalent malignancy and the sixth leading cause of cancer-associated mortality in the global population $(2,3)$. A number of studies have been performed with regard to prostate cancer, but the pathogenesis of the disease remains unclear. The established prognostic factors for prostate cancer are the Gleason score (GS), pathological T (pT) staging and serum prostatic-specific antigen (PSA) level (4). Ki-67 and Myc proto-oncogene protein have also been documented as prognostic factors for biochemical recurrence (BCR), metastatic disease and cancer-related mortality $(5,6)$. However, these prognostic factors alone are not sufficient for predicting prognostic characteristics, including early stage or advanced prostate cancer, presence of metastasis or disease-related mortality. Thus, novel prognostic biomarkers have been suggested for prostate cancer management. Recently, biomarkers such as transcriptional regulator ERG (ERG), phosphatase and tensin homolog (PTEN), cysteine-rich secretory protein 3 (CRISP3) and serine protease inhibitor Kazal type I (SPINK1) have been considered useful prognostic factors, although contradictory results have been obtained with regard to BCR and cancer-related mortality. The mutual associations of these biomarkers remain to be verified, as the four biomarkers have not previously been studied together.

The purpose of the present study was to simultaneously evaluate the prognostic value and associations of four biomarkers, namely, ERG, PTEN, CRISP3 and SPINK1, and to conduct risk stratification of prostate cancer for patient management. This study is the first to research ERG, PTEN, CRISP3 and SPINK1 expression concurrently.

\section{Materials and methods}

Clinical study samples and assessment. The use of clinical tumor samples was approved by the Institutional Review Board of the Kyung Hee University Hospital (Seoul, Korea). A total of 68 formalin-fixed, paraffin-embedded, prostate cancer samples from radical prostatectomies performed between January 2004 and December 2012, were obtained from Kyung Hee University 
Medical Center. The mean follow-up time was 49.2 months (range, 11-111 months). At the time of tumor sampling, none of the patients had been administered previous radiation therapy or chemotherapy, and there was no evidence of metastasis.

For all microscopy experiments, a BX53 microscope (Olympus Corporation, Tokyo, Japan) was used. The samples were reviewed by three pathologists from the hospital according to the pathological T (pT) staging of the American Joint Committee on Cancer 2010 criteria (7th edition) (7) and GS grading according to the International Society of Urological Pathology 2005 criteria (8). GSs were grouped as values of $<7$, equal to 7 , or $>7$ (3-tiered system). GSs of $3+4$ and $4+3$ were combined (9). The demographics of the tumor samples are shown in Table I.

Following histological diagnosis, PSA tests were conducted. A PSA level with a second confirmatory level $>0.2 \mathrm{ng} / \mathrm{ml}$ was defined as BCR (10). The period from the time of surgical treatment to BCR represents the BCR-free survival time.

Immunohistochemistry. Sections (5- $\mu \mathrm{m}$ thick) were cut from the formalin-fixed, paraffin-embedded tissue and stained with Leica auto-stainer Bond Max using the Bond Polymer Refine Detection System (Leica Biosystems Newcastle Ltd., Newcastle, UK) according to the manufacturer's protocols, with minor modifications. Briefly, the sections were deparaffinized by Bond Dewax Solution (Leica Biosystems Newcastle Ltd.), followed by heat-induced antigen retrieval using Bond Epitope retrieval solution 1 (for SPINK1) or 2 (for ERG, PTEN and CRISP3) (Leica Biosystems Newcastle Ltd.) for $20 \mathrm{~min}$ at $100^{\circ} \mathrm{C}$. The endogenous peroxidase was quenched by incubation with hydrogen peroxide for $15 \mathrm{~min}$. Sections were incubated for $15 \mathrm{~min}$ at ambient temperature with mouse monoclonal ERG (catalog. no. CM 421 A, C; Biocare Medical LLC, Concord, CA, USA) at a 1:100 dilution, with mouse monoclonal PTEN (CM 278 AK, CK; Biocare Medical LLC) at a 1:100 dilution, with rabbit polyclonal antibody CRISP3 (LS-B8285; LifeSpan Biosciences Inc., Seattle, WA, USA) at a 1:500 dilution, and with mouse monoclonal SPINK1 (ab58227; Abcam, Cambridge, MA, USA) at a 1:1,000 dilution. Bound primary antibodies were visualized using a biotin-free polymeric horseradish peroxidase-linker antibody conjugate system in a Bond-Max automatic slide stainer (Leica Biosystems Melbourne Pty. Ltd., Melbourne, Victoria, Australia). The nuclei of these sections were counterstained with hematoxylin in the Bond Polymer detection kit (Leica Biosystems, Newcastle, UK). Vascular endothelial cells for ERG and normal benign prostate gland and stroma cells for PTEN were based on healthy sites in the present patients and used as a positive internal control. Normal pancreatic tissues for CRISP3 and SPINK1 were obtained from the remaining tissues of patients that underwent a pancreatectomy due to chronic pancreatitis and used as an external positive control.

Pathological analysis of immunohistochemistry. Immunohistochemistry results were assessed and scored by the intensity and proportion (fraction) of positive tumor cells. The staining intensity was graded as follows (4-tiered system): No staining, 0 ; only visible at high magnification, $1+$; visible at low magnification, 2+; and striking at low magnification, 3+.
Table I. Demographics of the prostate cancer samples.

\begin{tabular}{lr} 
Clinicopathological parameters & Value \\
\hline Age in years, $\mathrm{n}(\%)$ & \\
$<50$ & $9(13.2)$ \\
$50-59$ & $38(55.9)$ \\
$>60$ & $21(30.9)$ \\
Pretreatment PSA in $\mathrm{ng} / \mathrm{ml}, \mathrm{n}(\%)$ & \\
$<4$ & $8(11.8)$ \\
$4-10$ & $31(45.6)$ \\
$10-20$ & $18(26.5)$ \\
$>20$ & $5(7.4)$
\end{tabular}

Tumor volume $\%, \mathrm{n}(\%)$

$<5$

$5-10$

$13(19.1)$

$10-20$

$12(17.6)$

$20-50$

$14(20.6)$

$>50$

Gleason score, n (\%)

$<7$

19 (27.9)

$=7$

$36(52.9)$

$>7$

pT stage ${ }^{\mathrm{a}}, \mathrm{n}(\%)$

pT2

$38(55.9)$

pT3

$30(44.1)$

Surgical margin, n (\%)

Negative

Positive

Lymphovascular invasion, n (\%)

Absent

$54(79.4)$

Present

Perineural invasion, n (\%)

Absent

$37(54.4)$

Present

$31(45.6)$

Metastasis, n (\%)

Absent

Present

Biochemical recurrence, n (\%)

Absent

Present

Mortality, n (\%)

Alive

Succumbed

Follow-up, months

Range

Mean

49.2

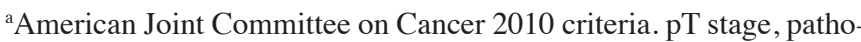
logical T stage; PSA, prostatic-specific antigen.

The final score for ERG expression was based on nuclear staining and was classified as follows (11): Negative, absence 
A ERG expression
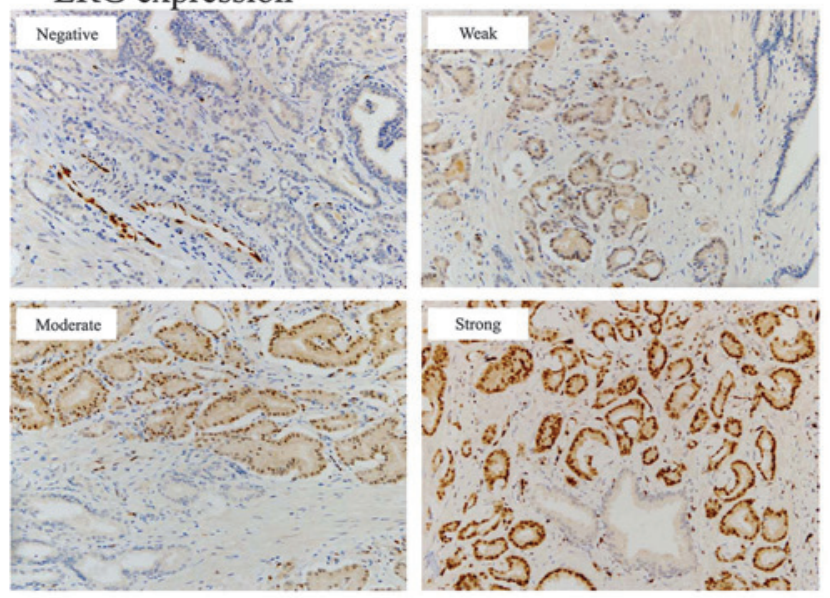

C CRISP3 expression
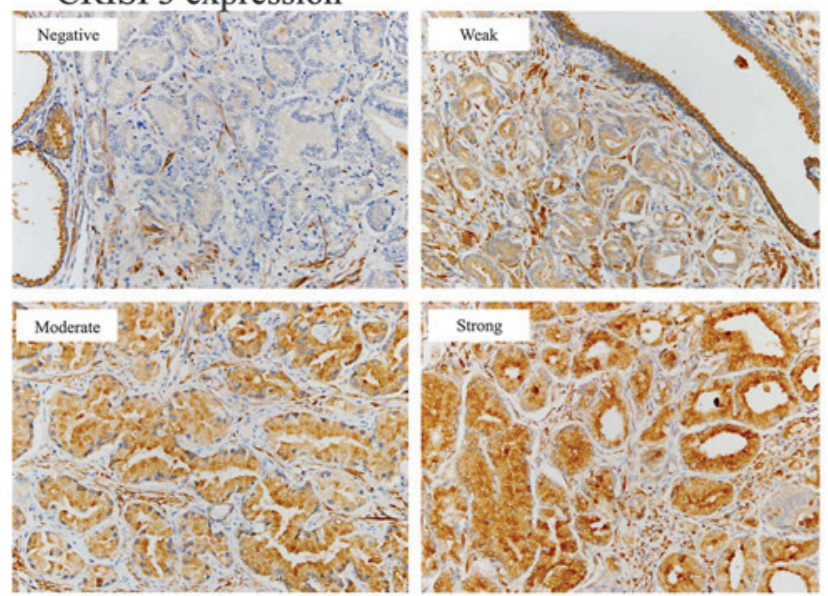

\section{B PTEN expression}

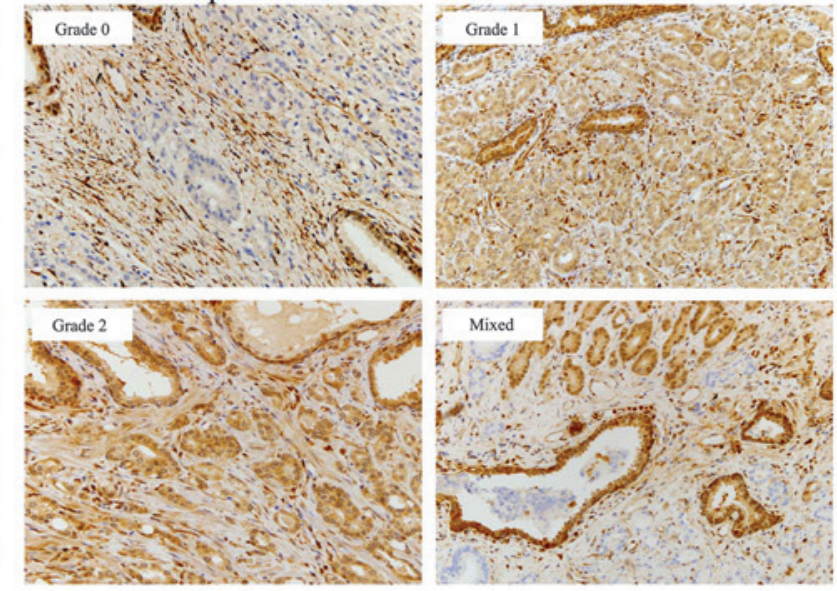

\section{SPINK1 expression}

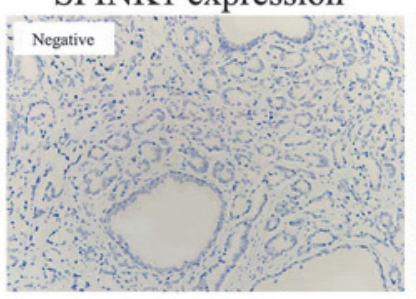

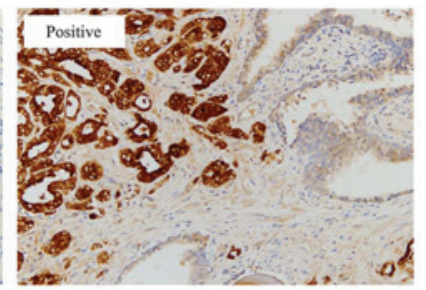

Figure 1. Immunohistochemical analysis of ERG, PTEN, CRISP3 and SPINK1. Original magnification, x200. (A) ERG expression is based on nuclear staining and classified as negative, weak, moderate and strong. (B) PTEN expression shows cytoplasmic staining classified as a 3-tiered system, and a mixed pattern is also noted. (C) CRISP3 expression is evaluated as cytoplasmic staining categorized as negative, weak, moderate and strong. (D) SPINK1 expression is assessed as negative and positive cytoplasmic staining. ERG, transcriptional regulator ERG; PTEN, phosphatase and tensin homolog; CRISP3, cysteine-rich secretory protein 3; SPINK1, serine protease inhibitor Kazal type I.

of ERG-stained cells; weak, intensity 1+ nuclear staining; moderate, intensity $2+$ nuclear staining; and strong, intensity $3+$ nuclear staining (Fig. 1A). Only weak to strong staining was considered as positive for ERG.

The immunohistochemical scoring of PTEN staining was based on cytoplasmic staining as part of a 3-tiered system as follows (12): Grade 0, absence of PTEN-positive tumor cells; grade 1, less staining than that of the normal prostate gland tissue; and grade 2, equal to or stronger staining of the normal prostate gland tissue (Fig. 1B).

Next, a final score for CRISP3 was evaluated according to cytoplasmic staining and was categorized as negative, weak, moderate or strong using the intensity score and proportion score of positive cells, as previously documented $(13,14)$ : Negative, absence of CRISP3 staining in 100\% of tumor cells; weak, intensity $1+$ in $>70 \%$ of tumor cells, or intensity $2+$ in $\leq 30 \%$ of tumor cells; moderate, intensity $1+$ in $>70 \%$ of tumor cells, intensity $2+$ in $>30$ but $\leq 70 \%$ of tumor cells, or intensity $3+$ in $\leq 30 \%$ of tumor cells; strong, intensity $2+$ in $>70 \%$ of tumor cells or intensity of $3+$ in $>30 \%$ of tumor cells (Fig. 1C).

Cytoplasmic staining was also classified into negative and positive SPINK1 expression as follows (2-tiered system) (15):
Negative, $\leq 5 \%$ stained cells; and positive, $>5 \%$ stained cells (Fig. 1D).

Analyses were performed for the correlations of the two biomarkers (PTEN, CRISP3) with clinicopathological parameters, BCR and cancer-related mortalities using a binary system approach, grouping low expression (negative/weak for CRISP3; and grade 0/grade 1 for PTEN) vs. high expression (moderate/strong for CRISP3; and grade 2 for PTEN).

Statistical analysis. Statistical analyses were performed using SPSS version 12.0 (IBM SPSS, Armonk, NY, USA). Pearson's $\chi^{2}$ test, Fisher's exact test and Student's t-test were conducted to determine correlations between the tested antibodies and clinicopathological parameters. Univariate survival analyses were performed to examine the prognostic significance of antibody expression and clinicopathological parameters, according to the Kaplan-Meier curve with a log-rank test. Multivariate survival analysis was conducted for all clinicopathological parameters and biomarkers, on the basis of the Cox proportional hazards model (95\% confidence interval) with the forward stepwise elimination method. $\mathrm{P}<0.05$ was considered to indicate a statistically significant difference. 


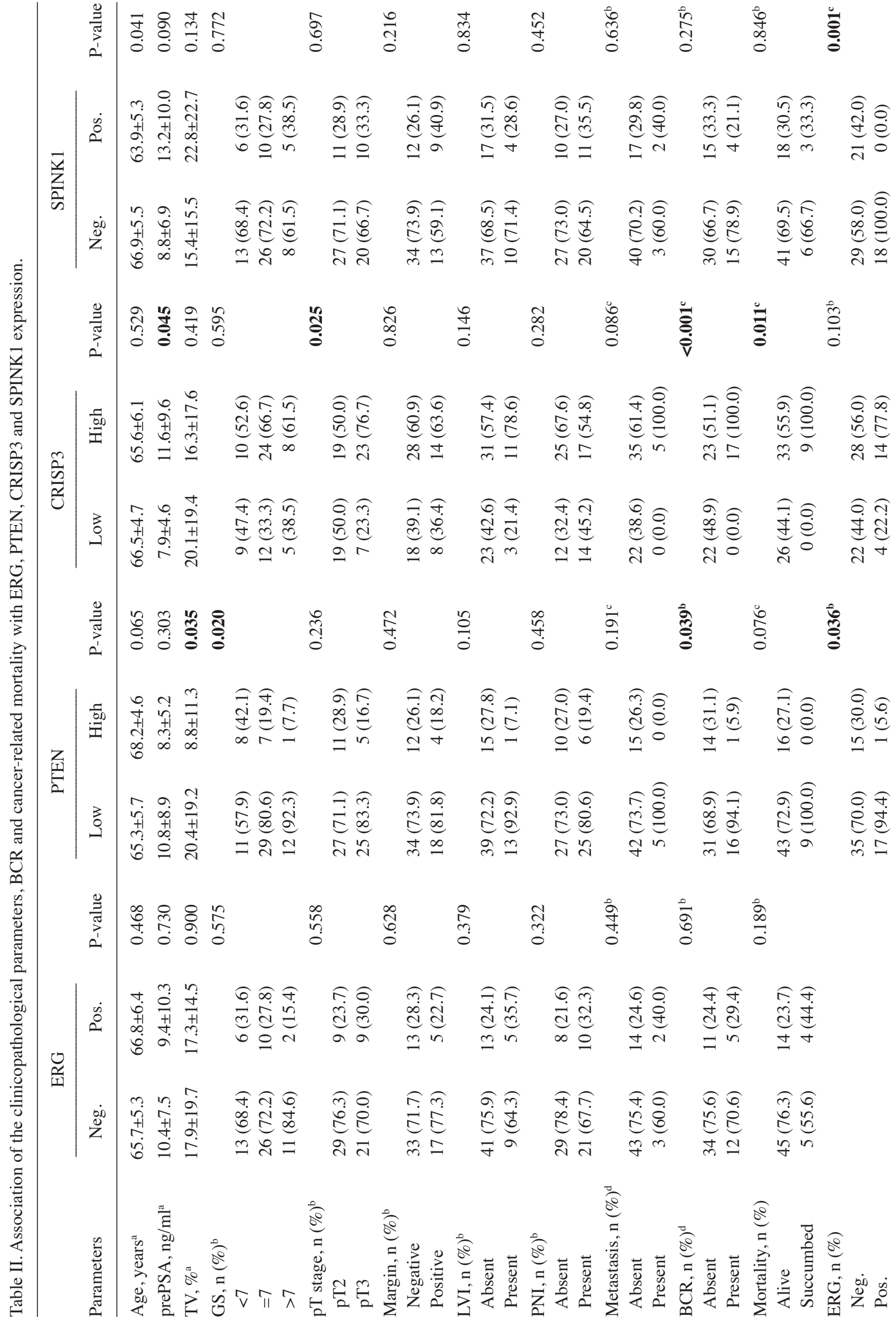




\section{Results}

Frequency of ERG, PTEN, CRISP3 and SPINK1 expression. From the tumor samples, positive ERG, low PTEN, high CRISP3 and positive SPINK1 expression was found in $18 / 68$ (26.5\%), 52/68 (76.5\%), $42 / 68(61.8 \%)$ and 21/68 (30.9\%) samples, respectively.

Associations between ERG, PTEN, CRISP3 and SPINK1 expression, and clinicopathological parameters

ERG expression. There was a non-significant trend between positive ERG expression and a GS of $<7$. Positive ERG expression in patients with a GS of $<7$ was noted in 6/19 samples (31.6\%) compared with $12 / 49$ samples $(24.5 \%)$ in patients with a GS of $\geq 7$ ( $\mathrm{P}=0.575$; Table II).

PTEN expression. Significant associations existed between low PTEN expression and a high tumor volume and a high GS; low PTEN expression was associated with a significant increase in tumor volume $(20.4 \pm 19.2 \%)$, compared with high PTEN expression $(8.8 \pm 11.3 \%)(\mathrm{P}=0.035$; Table II). Low PTEN in patients with a GS of $\geq 7$ was expressed in $41 / 49$ samples $(83.7 \%)$ vs. $11 / 19$ samples $(57.9 \%)$ in those with a GS of $<7$ $(\mathrm{P}=0.020$; Table II).

It was observed that low PTEN expression occurred more frequently in prostate cancers with lymphovascular invasion (LVI), high pT stage, high pre-treatment PSA (prePSA) level, metastasis or cancer-related mortality, although the differences were not significant. Low PTEN expression occurred in 13/14 samples (92.9\%) with LVI vs. 39/54 samples (72.2\%) without LVI ( $\mathrm{P}=0.105)$; in $25 / 30$ samples $(83.3 \%)$ in pT3 vs. $27 / 38$ samples $(71.1 \%)$ in pT2 ( $\mathrm{P}=0.236)$; in $5 / 5$ samples (100.0\%) with metastasis vs. $42 / 57$ samples $(73.7 \%)$ without metastasis $(\mathrm{P}=0.191)$; and in $9 / 9$ samples $(100.0 \%)$ in decedents vs. $43 / 59$ samples (72.9\%) in living patients $(\mathrm{P}=0.076)$ (Table II). There was also a non-significant increase in prePSA $(10.8 \pm 8.9 \mathrm{ng} / \mathrm{ml})$ in samples with low PTEN, compared with samples $(8.3 \pm 5.2 \mathrm{ng} / \mathrm{ml})$ with high PTEN expression $(\mathrm{P}=0.303$; Table II).

CRISP3 expression. High CRISP3 expression was significantly correlated with high prePSA level and high pT stage; CRISP3 overexpression demonstrated a significant increase in prePSA $(11.6 \pm 9.6 \mathrm{ng} / \mathrm{ml})$ compared with low CRISP3 expression $(7.9 \pm 4.6 \mathrm{ng} / \mathrm{ml})(\mathrm{P}=0.045$; Table II). CRISP3 overexpression in pT3 was identified in $23 / 30$ samples $(76.7 \%)$ vs. $19 / 38$ samples $(50.0 \%)$ in pT2 $(\mathrm{P}=0.025$; Table II).

High CRISP3 expression was detected more frequently in prostate cancers with LVI than in those without, although the difference was not statistically significant; high CRISP3 expression was noted in $11 / 14$ samples $(78.6 \%)$ with LVI vs. 31/54 samples (57.4\%) without LVI ( $\mathrm{P}=0.146$; Table II).

SPINK1 expression. It was found that prostate cancers that were positive for SPINK1 expression tended to have high prePSA levels compared with those without SPINK1 expression, although the difference was not significant; an increase in prePSA $(13.2 \pm 10.0 \mathrm{ng} / \mathrm{ml})$ was noted in cancers that overexpressed SPINK1 compared with those that were negative for SPINK1 expression $(8.8 \pm 6.9 \mathrm{ng} / \mathrm{ml})(\mathrm{P}=0.09$; Table II). SPINK1 was not associated with any of the clinicopathological parameters. 
Table III. Associations of the clinicopathological parameters with the low- and high-risk subgroups.

\begin{tabular}{|c|c|c|c|}
\hline Parameters & Low-risk $^{\mathrm{a}}$ & High-risk $^{\mathrm{b}}$ & P-value \\
\hline Age, years $^{c}$ & $67.0 \pm 4.9$ & $64.8 \pm 6.0$ & 0.101 \\
\hline prePSA, ng/ml ${ }^{\mathrm{c}}$ & $7.9 \pm 4.7$ & $12.7 \pm 10.4$ & 0.027 \\
\hline Tumor volume, $\%^{\mathrm{c}}$ & $16.1 \pm 18.3$ & $19.5 \pm 18.5$ & 0.468 \\
\hline Gleason score, $\mathrm{n}(\%)^{\mathrm{d}}$ & & & 0.069 \\
\hline$<7$ & $14(73.7)$ & $5(26.3)$ & \\
\hline$=7$ & $16(44.4)$ & $20(55.6)$ & \\
\hline$>7$ & $5(38.5)$ & $8(61.5)$ & \\
\hline pT stage, $\mathrm{n}(\%)^{\mathrm{d}}$ & & & 0.002 \\
\hline pT2 & $26(68.4)$ & $12(31.6)$ & \\
\hline pT3 & $9(30.0)$ & $21(70.0)$ & \\
\hline Surgical margin, $\mathrm{n}(\%)^{\mathrm{d}}$ & & & 0.867 \\
\hline Negative & $24(52.2)$ & $22(47.8)$ & \\
\hline Positive & $11(50.0)$ & $11(50.0)$ & \\
\hline LVI, n $(\%)^{\mathrm{d}}$ & & & 0.012 \\
\hline Absent & $32(59.3)$ & $22(40.7)$ & \\
\hline Present & $3(21.4)$ & $11(78.6)$ & \\
\hline PNI, n $(\%)^{\mathrm{d}}$ & & & 0.983 \\
\hline Absent & $19(51.4)$ & $18(48.6)$ & \\
\hline Present & $16(51.6)$ & $15(48.4)$ & \\
\hline Metastasis, $\mathrm{n}(\%)^{\mathrm{e}}$ & & & 0.021 \\
\hline Absent & $31(54.4)$ & $26(45.6)$ & \\
\hline Present & $0(0.0)$ & $5(100.0)$ & \\
\hline $\mathrm{BCR}, \mathrm{n}(\%)^{\mathrm{d}}$ & & & $<0.001$ \\
\hline Absent & $30(66.7)$ & $15(33.3)$ & \\
\hline Present & $1(5.3)$ & $18(94.7)$ & \\
\hline Mortality, n $(\%)^{\mathrm{e}}$ & & & 0.001 \\
\hline Alive & $35(59.3)$ & $24(40.7)$ & \\
\hline Dead & $0(0.0)$ & $9(100.0)$ & \\
\hline
\end{tabular}

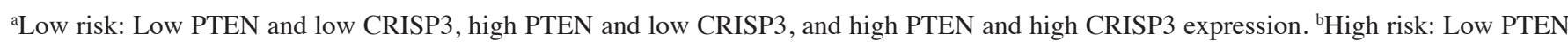
and high CRISP3 expression. ${ }^{\mathrm{c}}$ Student's t-test, data are presented as mean \pm standard deviation. ${ }^{\mathrm{d}} \chi^{2}$ test. ${ }^{\mathrm{e}}$ Fisher's exact test. BCR, biochemical recurrence; CRISP3, cysteine-rich secretory protein 3; PNI, perineural invasion; prePSA, pretreatment prostate-specific antigen; PTEN, phosphatase and tensin homolog; LVI, lymphovascular invasion.

Table IV. Multivariate analysis (Cox's proportional hazard model).

\begin{tabular}{|c|c|c|c|c|c|c|}
\hline \multirow[b]{2}{*}{ Parameter } & \multirow[b]{2}{*}{$\mathrm{B}$} & \multirow[b]{2}{*}{ SE } & \multirow[b]{2}{*}{ P-value } & \multirow[b]{2}{*}{ HR } & \multicolumn{2}{|c|}{$95 \% \mathrm{CI}$} \\
\hline & & & & & Lower & Upper \\
\hline Subgroup, high-risk ${ }^{\mathrm{a}}$ vs. low-risk ${ }^{\mathrm{b}}$ & 2.300 & 1.062 & 0.030 & 9.979 & 1.244 & 80.031 \\
\hline pT stage, pT3 vs. pT2 & 2.040 & 1.048 & 0.052 & 7.687 & 0.985 & 59.996 \\
\hline Metastasis, present vs. absent & 1.013 & 0.586 & 0.084 & 2.754 & 0.873 & 8.682 \\
\hline
\end{tabular}

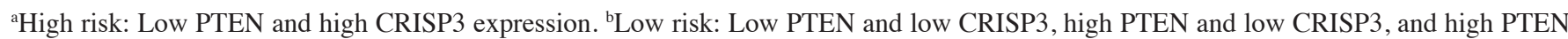
and high CRISP3 expression. B, regression coefficient; CI, confidence interval; HR, hazard ratio; SE, standard error; pT, pathological T stage.

Associations between ERG, PTEN, CRISP3 and SPINKI expression. A significant inverse association between ERG, PTEN and SPINK1 expression was verified in the present study.
Low PTEN expression in prostate cancer with positive ERG expression was noted in 17/18 samples (94.4\%) vs. 35/50 samples (70.0\%) with negative ERG expression ( $\mathrm{P}=0.036$; Table II). 

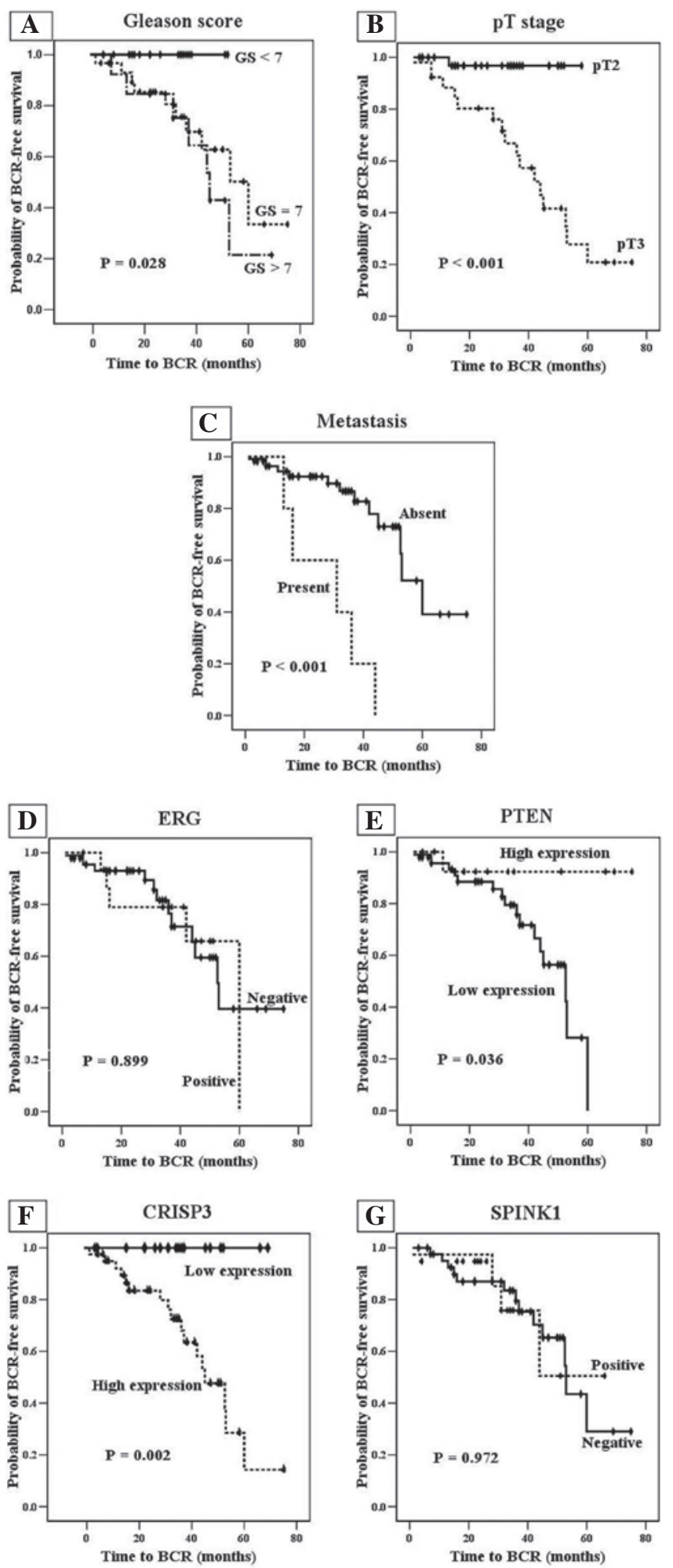

Figure 2. Univariate analysis (Kaplan-Meier curves) of biochemical recurrence (BCR)-free survival in association with (A) Gleason score, (B) pathological tumor (pT) stage and (C) metastasis, and expression of (D) transcriptional regulator ERG (ERG), (E) phosphatase and tensin homolog (PTEN), (F) cysteine-rich secretory protein 3 (CRISP3) and (G) serine protease inhibitor Kazal type I (SPINK1).

SPINK1 expression was inversely associated with ERG expression; negative SPINK1 expression in tumor samples with positive ERG expression was detected in 18/18 (100.0\%) vs. 29/50 samples (58.0\%) with negative ERG expression ( $\mathrm{P}=0.001$; Table II).
Low and high-risk subgroups

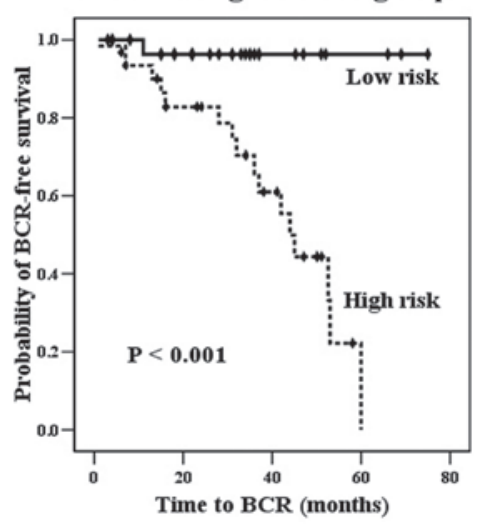

Figure 3. Univariate analysis (Kaplan-Meier curve) for low-risk and high-risk subgroups. BCR, biochemical recurrence.

CRISP3 was overexpressed in ERG-positive prostate cancers, but with a non-significant positive trend; high CRISP3 expression in ERG-positive prostate cancer was shown in $14 / 18$ samples $(77.8 \%)$ vs. $28 / 50$ samples $(56.0 \%)$ in ERG-negative prostate cancer $(\mathrm{P}=0.103$; Table II).

Analysis of BCR-free survival. A shorter BCR-free survival time was significantly associated with high GS $(\mathrm{P}=0.028)$, high pT stage $(\mathrm{P}<0.001)$ and metastasis $(\mathrm{P}<0.001)$ among the clinicopathological parameters (Fig. 2A-C) upon univariate analysis (Kaplan-Meier curve and log-rank test). The other clinicopathological parameters of surgical margin, lymphovascular invasion and perineural invasion exhibited no significant statistical value for BCR-free survival.

It was also demonstrated that low PTEN and high CRISP3 expression were associated with decreased BCR-free survival $(\mathrm{P}=0.036$ and $\mathrm{P}=0.002$, respectively; Fig. $2 \mathrm{E}$ and $\mathrm{F})$ upon univariate analysis. Positive ERG expression was not significantly associated with BCR-free survival ( $\mathrm{P}=0.889$; Fig. 2D). Therefore, as positive ERG expression was not associated with BCR compared with low PTEN or high CRISP3 expression, this strengthened the hypothesis that positive ERG expression is present in early-stage prostate cancer $(16,17)$. However, the PTEN and CRISP3 biomarkers did not exhibit prognostic value for BCR-free survival upon the multivariate analysis (Cox's proportional hazard model).

Defining low- and high-risk subgroups, and correlation of subgroups with BCR. On the basis of the aforementioned results, low-risk and high-risk subgroups were created; the high-risk subgroup was defined as prostate cancer cases with low PTEN and high CRISP3 expression, and the low-risk subgroup was defined as prostate cancer cases with low PTEN and low CRISP3, high PTEN and low CRISP3, or high PTEN and high CRISP3 expression. The association of the low- and high-risk subgroups with clinicopathological parameters, BCR and cancer-related mortality is demonstrated in Table III. The high-risk prostate cancers were significantly correlated with high prePSA, high GS, high pT stage, LVI, metastasis, BCR and cancer-related mortality. The high-risk subgroup showed 28/49 samples (57.1\%) with a GS of $\geq 7$ vs. $5 / 19$ samples $(26.3 \%)$ with a $\mathrm{GS}$ of $<7(\mathrm{P}=0.022)$; 
21/30 samples (70.0\%) in pT3 vs. $12 / 38$ samples $(31.6 \%)$ in pT2 $(\mathrm{P}=0.002) ; 11 / 14$ samples (78.6\%) with LVI vs. 22/54 samples (40.7\%) without LVI $(\mathrm{P}=0.012)$; $5 / 5$ samples $(100.0 \%)$ with metastasis vs. $26 / 57$ samples $(45.6 \%)$ without metastasis $(\mathrm{P}=0.021) ; 18 / 19$ samples $(94.7 \%)$ with BCR vs. $15 / 45$ samples (33.3\%) without BCR $(\mathrm{P}<0.001)$; and 9/9 samples $(100.0 \%)$ in decedents vs. $24 / 59$ samples $(40.7 \%)$ in living patients $(\mathrm{P}=0.001)$. The high-risk subgroup also presented with significantly increased prePSA levels $(12.7 \pm 10.4 \mathrm{ng} / \mathrm{ml})$ compared with those of the low-risk subgroup $(7.9 \pm 4.7 \mathrm{ng} / \mathrm{ml})(\mathrm{P}=0.027)$ (Table III).

The high-risk subgroup with low PTEN and high CRISP3 expression was shown to be significantly predictive of the prognosis for BCR-free survival using univariate analysis $(\mathrm{P}<0.001$; Fig. 3) and multivariate analysis ( $\mathrm{P}=0.030$; Table IV). The other factors, such as high pT stage (pT3) and metastasis, showed unfavorable trends for shorter $\mathrm{BCR}(\mathrm{P}=0.052$ and $\mathrm{P}=0.084$, respectively), but these differences were not significant.

\section{Discussion}

The prevalence of transmembrane protease serine 2 (TMPRSS2):ERG fusion in prostate cancer depends on genetic and ethnic differences, with a much higher prevalence in Caucasian individuals: $36-60 \%$ in USA, Germany and Canada; $48 \%$ in Brazil; 20.9-26.5\% in Korea (including the present study); $23.2 \%$ in China; and 28\% in Japan $(1,12,18)$.

TMPRSS2:ERG rearrangement in prostate cancer was initially demonstrated by the microdeletion of chromosome 21 and the insertion of the deleted region into a different chromosome, resulting in TMPRSS2:ERG fusion (19). This fusion between the androgen-driven gene, TMPRSS2, and the oncogenic transcription factor genes of the E26 transformation-specific (ETS) family has been recognized as a critical event. Presently, 14 5' fusion partners (TMPRSS2, SLC45A3, HERV-K, KLK2, CANT1, FOXP1, HERVK17, EST14, ELJ35294, C15orf21, HNRPA2B1, DDX5, ACSL3 and NDRG1) and 4 ETS family genes (ERG, ETV1, ETV4 and ETV5) have been found, and the TMPSS2:ERG fusion accounts for $\sim 90 \%$ of all fusions (18). The majority of ETS family transcription factor genes have an 85-amino acid ETS domain that promotes binding to the specific GGAA/T core consensus sequence and regulates the expression of target genes associated with prostate cancer generation and progression (20). The TMPRSS2:ERG fusion protein is detected by immunohistochemistry, as ERG overexpression by immunohistochemistry is highly concordant with ERG fusion or rearrangement by reverse transcription-quantitative polymerase chain reaction or fluorescence in situ hybridization $(21,22)$.

The PTEN gene on chromosome 10q23 is a tumor suppressor gene, and PTEN loss releases the regulation of the receptor tyrosine kinase (RTK)/phosphatidylinositol 3-kinase (PI3K)/Akt signaling pathway. PTEN loss promotes prostate cancer progression and metastasis, and is present in aggressive and hormone-refractory prostate cancer (23-28). The RTK/PI3K/Akt pathway has also been linked to cholesterol ester accumulation and chemokine (C-X-C motif) ligand 12/chemokine (C-X-C motif) receptor $4(29,30)$. PTEN genomic loss (heterozygous or homozygous PTEN deletion) has been detected with an incidence of 75-86\% PTEN protein loss. PTEN protein loss is significantly associated with PTEN genomic loss $(4,28)$.

CRISP3 consists of 254 amino acids and is encoded by a locus on 6p12.3. CRISP3 is an extracellular matrix protein found in the salivary glands and male reproductive tract, and its expression is driven by androgens. CRISP 3 is intracellularly stored in the granules of neutrophils and eosinophils, in either a glycosylated or unglycosylated form, and is secreted for the innate host defense, resulting in cellular matrix remodeling by proteolysis (31-33). The exact function of CRISP3 requires further research.

SPINK1 is a 56-amino acid extracellular secreted protein known as a pancreatic secretory trypsin inhibitor or a tumor-associated trypsin inhibitor (34). SPINK1 has been detected in benign lesions and malignancies, such as colorectal, hepatocellular and breast cancer (35-38). SPINK1 in prostate cancer can induce epithelial-mesenchymal transition through the epidermal growth factor receptor (EGFR)/mitogen-activated protein kinase (MAPK)/MAPK kinase/extracellular signal-regulated kinase signaling pathway (34).

It has been documented that ERG fusion is enriched for PTEN deletion (12), which is consistent with the findings from the present study. PTEN loss is significantly associated with an underlying ERG rearrangement (39), as ERG fusion can increase the expression of nuclear factor- $\mathrm{\kappa B}$ (regulator of PTEN transcription), inhibit PTEN transcription and manipulate the microenvironment to induce the chromosomal rearrangement of PTEN $(20,25)$.

ERG fusion expression is mutually exclusive of SPINK1 expression (15). SPINK1 overexpression is linked to prostate cancer with 6q15-and 5q21-deleted ERG fusion-negative genetic rearrangements (40).

It has been reported that CRISP3 protein overexpression is significantly correlated with ERG fusion-positive prostate cancers $(33,41)$. CRISP3 is a direct target of member of the ETS family, such as ERG, as it has a specific GGAA/T core consensus sequence (33).

One clinicopathological parameter that has been associated with ERG overexpression is low GS $(<7)(1,18)$; however, the correlation between BCR or cancer-related mortality and ERG overexpression has shown contradictory results in previous studies $(12,42,43)$. In the present study, ERG overexpression was not significantly associated with a low GS or cancer-related mortality. Notably, certain studies have demonstrated that ERG fusion tends to occur in early-stage prostate cancer and can be bypassed at late-stage androgen-refractory prostate cancer $(16,17)$.

It has been revealed that PTEN loss is significantly associated with a high GS, advanced pT stage, p53 accumulation $(23,28), B C R$ and cancer-related mortality $(4,23)$, while CRISP3 overexpression is also associated with a high GS, BCR and cancer-related mortality $(13,14,33,41)$, as shown in the present study.

It has been documented that there is a significant association between SPINK1 overexpression and BCR or cancer-related mortality, although this association was not significant in a few studies (including the present study) (44). The present study showed that a high PSA level was not significantly associated 
with high SPINK1 expression ( $\mathrm{P}=0.09$; Table II). Based on this result, we speculate that SPINK1 overexpression decreases BCR-free survival.

Briefly, ERG fusion is central in the regulation of PTEN, CRISP3 and SPINK1 expression. This fusion is a pathognomonically important event in prostate cancer pathogenesis. PTEN loss and increased CRISP3 expression can be promoted by positive ERG expression. SPINK1 expression is mutually exclusive of ERG expression.

PTEN loss and the high expression of CRISP3 can be unfavorable prostate cancer indicators, as PTEN loss is associated with a shorter BCR and with hormone-refractory prostate cancer. A high level of CRISP3 expression also increases BCR and cancer-related mortality. The loss of PTEN or CRISP3 overexpression may be used as a better predictor of progressed (advanced) prostate cancer than ERG fusion expression, although is does not provide significant results for BCR. However, a single biomarker of either PTEN or CRISP3 is insufficient to predict the prognosis for BCR. For this reason, combined PTEN and CRISP3 expression may be used with regard to patients with a poor prognosis. Categorizing patients into high- and low-risk subgroups is well correlated with BCR-free survival in prostate cancer, as aforementioned in the present study (Table III; Fig. 3).

In conclusion, the present study suggests that low PTEN expression and high CRISP3 expression significantly characterize the subgroups of prostate cancer with a poor prognosis for BCR. These results will aid in the management and stratification of patients with advanced prostate cancer.

\section{Acknowledgements}

The abstract was presented at the United States \& Canadian Academy of Pathology 104th Annual Meeting Abstracts and published in Modern Pathol (Suppl 2s): 247A-248A, 2015.

\section{References}

1. Lee K, Chae JY, Kwak C, Ku JH and Moon KC: TMPRSS2-ERG gene fusion and clinicopathologic characteristics of Korean prostate cancer patients. Urology 76: 1268.e7-1268.e13, 2010.

2. Jemal A, Bray F, Center MM, Ferlay J, Ward E and Forman D: Global cancer statistics. CA Cancer J Clin 61: 69-90, 2011.

3. Siegel R, Ma J, Zou Z and Jemal A: Cancer statistics, 2014. CA Cancer J Clin 64: 9-29, 2014

4. Cuzick J, Yang ZH, Fisher G, Tikishvili E, Stone S, Lanchbury JS, Camacho N, Merson S, Brewer D, Cooper CS, et al: Prognostic value of PTEN loss in men with conservatively managed localised prostate cancer. Br J Cancer 108: 2582-2589, 2013.

5. Liu W, Xie CC, Thomas CY, Kim ST, Lindberg J, Egevad L, Wang Z, Zhang Z, Sun J, Sun J, et al: Genetic markers associated with early cancer-specific mortality following prostatectomy. Cancer 119: 2405-2412, 2013.

6. Van der Kwast TH: Prognostic prostate tissue biomarkers of potential clinical use. Virchows Arch 464: 293-300, 2014.

7. Edge S, Byrd DR, Compton CC, Fritz AG, Greene FL and Trotti A: AJCC Cancer Staging Handbook. 7th edition. Springer-Verlag, New York, NY, 2010.

8. Epstein JI, Allsbrook WC Jr, Amin MB and Egevad LL; ISUP Grading Committee: The 2005 international society of urological pathology (ISUP) consensus conference on gleason grading of prostatic carcinoma. Am J Surg Pathol 29: 1228-1242, 2005.

9. Cuzick J, Fisher G, Kattan MW, Berney D, Oliver T, Foster CS, Møller H, Reuter V, Fearn P, Eastham J, et al: Long-term outcome among men with conservatively treated localised prostate cancer. Br J Cancer 95: 1186-1194, 2006.
10. Punnen S, Cooperberg MR, D'Amico AV, Karakiewicz PI, Moul JW, Scher HI, Schlomm T and Freedland SJ: Management of biochemical recurrence after primary treatment of prostate cancer: A systematic review of the literature. Eur Urol 64: 905-915, 2013.

11. He H, Magi-Galluzzi C, Li J, Carver P, Falzarano S, Smith K, Rubin MA and Zhou M: The diagnostic utility of novel immunohistochemical marker ERG in the workup of prostate biopsies with 'atypical glands suspicious for cancer'. Am J Surg Pathol 35: 608-614, 2011.

12. Qi M, Yang X, Zhang F, Lin T, Sun X, Li Y, Yuan H, Ren Y, Zhang J, Qin X and Han B: ERG rearrangement is associated with prostate cancer-related death in Chinese prostate cancer patients. PLoS One 9: e84959, 2014.

13. Dahlman A, Rexhepaj E, Brennan DJ, Gallagher WM, Gaber A, Lindgren A, Jirström $\mathrm{K}$ and Bjartell A: Evaluation of the prognostic significance of MSMB and CRISP3 in prostate cancer using automated image analysis. Mod Pathol 24: 708-719, 2011.

14. Al Bashir S, Alshalalfa M, Hegazy SA, Dolph M, Donnelly B and Bismar TA: Cysteine- rich secretory protein 3 (CRISP3), ERG and PTEN define a molecular subtype of prostate cancer with implication to patients' prognosis. J Hematol Oncol 7: 21, 2014.

15. Bhalla R, Kunju LP, Tomlins SA, Christopherson K, Cortez C, Carskadon S, Siddiqui J, Park K, Mosquera JM, Pestano GA, et al: Novel dual-color immunohistochemical methods for detecting ERG-PTEN and ERG-SPINK1 status in prostate carcinoma. Mod Pathol 26: 835-848, 2013.

16. Hermans KG, van Marion R, van Dekken H, Jenster G, van Weerden WM and Trapman J: TMPRSS2:ERG fusion by translocation or interstitial deletion is highly relevant in androgen-dependent prostate cancer, but is bypassed in late-stage androgen receptor-negative prostate cancer. Cancer Res 66: 10658-10663, 2006.

17. Saramäki OR, Harjula AE, Martikainen PM, Vessella RL, Tammela TL and Visakorpi T: TMPRSS2:ERG fusion identifies a subgroup of prostate cancers with a favorable prognosis. Clin Cancer Res 14: 3395-3400, 2008.

18. Miyagi Y, Sasaki T, Fujinami K, Sano J, Senga Y, Miura T, Kameda Y, Sakuma Y, Nakamura Y, Harada M and Tsuchiya E: ETS family-associated gene fusions in Japanese prostate cancer: Analysis of 194 radical prostatectomy samples. Mod Pathol 23: 1492-1498, 2010.

19. Yoshimoto M, Joshua AM, Chilton-Macneill S, Bayani J, Selvarajah S, Evans AJ, Zielenska M and Squire JA: Three-color FISH analysis of TMPRSS2/ERG fusions in prostate cancer indicates that genomic microdeletion of chromosome 21 is associated with rearrangement. Neoplasia 8: 465-469, 2006.

20. Sreenath TL, Dobi A, Petrovics G and Srivastava S: Oncogenic activation of ERG: A predominant mechanism in prostate cancer. J Carcinog 10: 37, 2011.

21. Shah RB: Clinical applications of novel ERG immunohistochemistry in prostate cancer diagnosis and management. Adv Anat Pathol 20: 117-124, 2013.

22. Braun M, Goltz D, Shaikhibrahim Z, Vogel W, Böhm D, Scheble V, Sotlar K, Fend F, Tan SH, Dobi A, et al: ERG protein expression and genomic rearrangement status in primary and metastatic prostate cancer-a comparative study of two monoclonal antibodies. Prostate Cancer Prostatic Dis 15: 165-169, 2012.

23. Krohn A, Diedler T, Burkhardt L, Mayer PS, De Silva C, Meyer-Kornblum M, Kötschau D, Tennstedt P, Huang J, Gerhäuser C, et al: Genomic deletion of PTEN is associated with tumor progression and early PSA recurrence in ERG fusion-positive and fusion-negative prostate cancer. Am J Pathol 181: 401-412, 2012.

24. Shen MM and Abate-Shen C: Molecular genetics of prostate cancer: New prospects for old challenges. Genes Dev 24: 1967-2000, 2010.

25. Zhang S and Yu D: PI(3)king apart PTEN's role in cancer. Clin Cancer Res 16: 4325-4330, 2010

26. Mulholland DJ, Tran LM, Li Y, Cai H, Morim A, Wang S, Plaisier S, Garraway IP, Huang J, Graeber TG and Wu H: Cell autonomous role of PTEN in regulating castration-resistant prostate cancer growth. Cancer Cell 19: 792-804, 2011.

27. Tian L, Fang YX, Xue JL and Chen JZ: Four microRNAs promote prostate cell proliferation with regulation of PTEN and its downstream signals in vitro. PLoS One 8: e75885, 2013.

28. Lotan TL, Gurel B, Sutcliffe S, Esopi D, Liu W, Xu J, Hicks JL, Park BH, Humphreys E, Partin AW, et al: PTEN protein loss by immunostaining: Analytic validation and prognostic indicator for a high risk surgical cohort of prostate cancer patients. Clin Cancer Res 17: 6563-6573, 2011. 
29. Yue S, Li J, Lee SY, Lee HJ, Shao T, Song B, Cheng L, Masterson TA, Liu X, Ratliff TL and Cheng JX: Cholesteryl ester accumulation induced by PTEN loss and PI3K/AKT activation underlies human prostate cancer aggressiveness. Cell Metab 19: 393-406, 2014.

30. Conley-LaComb MK, Saliganan A, Kandagatla P, Chen YQ, Cher ML and Chinni SR: PTEN loss mediated Akt activation promotes prostate tumor growth and metastasis via CXCL12/CXCR4 signaling. Mol Cancer 12: 85, 2013.

31. Udby L, Calafat J, Sorensen OE, Borregaard N and Kjeldsen L: Identification of human cysteine-rich secretory protein 3 (CRISP-3) as a matrix protein in a subset of peroxidase-negative granules of neutrophils and in the granules of eosinophils. J Leukoc Biol 72: 462-469, 2002.

32. Udby L, Cowland JB, Johnsen AH, Sørensen OE, Borregaard N and Kjeldsen L: An ELISA for SGP28/CRISP-3, a cysteine-rich secretory protein in human neutrophils, plasma and exocrine secretions. J Immunol Methods 263: 43-55, 2002.

33. Ribeiro FR, Paulo P, Costa VL, Barros-Silva JD, Ramalho-Carvalho J, Jerónimo C, Henrique R, Lind GE, Skotheim RI, Lothe RA and Teixeira MR: Cysteine-rich secretory protein-3 (CRISP3) is strongly up-regulated in prostate carcinomas with the TMPRSS2-ERG fusion gene. PLoS One 6: e22317, 2011.

34. Wang C, Wang L, Su B, Lu N, Song J, Yang X, Fu W, Tan W and Han B: Serine protease inhibitor Kazal type 1 promotes epithelial-mesenchymal transition through EGFR signaling pathway in prostate cancer. Prostate 74: 689-701, 2014.

35. Lamontagne J, Pinkerton M, Block TM and Lu X: Hepatitis B and hepatitis $\mathrm{C}$ virus replication upregulates serine protease inhibitor Kazal, resulting in cellular resistance to serine protease-dependent apoptosis. J Virol 84: 907-917, 2010.

36. Higashiyama M, Monden T, Tomita N, Murotani M, Kawasaki Y, Morimoto H, Murata A, Shimano T, Ogawa M and Mori T: Expression of pancreatic secretory trypsin inhibitor (PSTI) in colorectal cancer. Br J Cancer 62: 954-958, 1990.

37. Lee YC, Pan HW, Peng SY, Lai PL, Kuo WS, Ou YH and Hsu HC: Overexpression of tumour-associated trypsin inhibitor (TATI) enhances tumour growth and is associated with portal vein invasion, early recurrence and a stage-independent prognostic factor of hepatocellular carcinoma. Eur J Cancer 43 736-744, 2007.
38. Soon WW, Miller LD, Black MA, Dalmasso C, Chan XB, Pang B, Ong CW, Salto-Tellez M, Desai KV and Liu ET: Combined genomic and phenotype screening reveals secretory factor SPINK1 as an invasion and survival factor associated with patient prognosis in breast cancer. EMBO Mol Med 3: 451-464, 2011.

39. Reid AH, Attard G, Brewer D, Miranda S, Riisnaes R, Clark J, Hylands L, Merson S, Vergis R, Jameson C, et al: Novel, gross chromosomal alterations involving PTEN cooperate with allelic loss in prostate cancer. Mod Pathol 25: 902-910, 2012

40. Grupp K, Diebel F, Sirma H, Simon R, Breitmeyer K, Steurer S, Hube-Magg C, Prien K, Pham T, Weigand P, et al: SPINK1 expression is tightly linked to 6q15- and 5q21-deleted ERG-fusion negative prostate cancers but unrelated to PSA recurrence. Prostate 73: 1690-1698, 2013.

41. Grupp K, Kohl S, Sirma H, Simon R, Steurer S, Becker A, Adam M, Izbicki J, Sauter G, Minner S, et al: Cysteine-rich secretory protein 3 overexpression is linked to a subset of PTEN-deleted ERG fusion-positive prostate cancers with early biochemical recurrence. Mod Pathol 26: 733-742, 2013.

42. Nam RK, Sugar L, Yang W, Srivastava S, Klotz LH, Yang LY, Stanimirovic A, Encioiu E, Neill M, Loblaw DA, et al: Expression of the TMPRSS2:ERG fusion gene predicts cancer recurrence after surgery for localised prostate cancer. $\mathrm{Br} \mathrm{J}$ Cancer 97: 1690-1695, 2007.

43. Dal Pra A, Lalonde E, Sykes J, Warde F, Ishkanian A, Meng A, Maloff C, Srigley J, Joshua AM, Petrovics G, et al: TMPRSS2-ERG status is not prognostic following prostate cancer radiotherapy: Implications for fusion status and DSB repair. Clin Cancer Res 19: 5202-5209, 2013.

44. Nagle RB, Algotar AM, Cortez CC, Smith K, Jones C, Sathyanarayana UG, Yun S, Riley J, Nagy D, Dittamore R, et al: ERG overexpression and PTEN status predict capsular penetration in prostate carcinoma. Prostate 73: 1233-1240, 2013. 